\title{
BITTORRENT SOFTWARE AND USAGE
}

\author{
Justin McKinney \\ Mark Simon Haydn
}

\section{Abstract:}

While the circulation of cultural material outside of official channels is not new, the scale and infrastructure afforded by digital networks and peer-to-peer protocols has drastically changed its dynamics. Focusing on private trackers and online, members-only communities dedicated to sharing difficult to find and "gray-area" cinema content, our paper discusses new digital repositories and their connection to the traditional film archive. With discussion of the types of materials held, user participation, and custodial efforts to restore or improve cultural material, we will discuss the activities of a contemporary private tracker community. Additionally, the paper will interrogate the legality and copyright issues surrounding these activities and explore recent, licit adoption of the infrastructure that has been developed for online circulation. Discussion will conclude with attention to cases in which pirated material has resurfaced in a rights-holding context, and an assessment of what these developments mean for custodians of film material working in a traditional film archive context. As a combination of discussion, case study, and argument, the paper will serve as a topical primer on a pressing and underresearched area of interest in this field, building on a panel presented at last year's Association of Moving Image Archivists conference in Georgia.

\section{BitTorrent Software and Usage}

BitTorrent is a software protocol developed in $200 \mathrm{I}$ and designed to aid the practice of peerto-peer file sharing on the Internet. The primary advantage of BitTorrent is that it allows for segmented downloading, which is the coordinated transmission of a file sourced from multiple servers to a single destination. ${ }^{81}$ This protocol allows for the rapid sharing of large amounts of data by allowing a user to download a file from multiple sources that are uploading the file at the same time. ${ }^{82}$ This allows for more efficient and faster downloading than the traditional client-server model. The primary impact of this has been the rise of file sharing of all forms of media including ebooks, audio files, software, and video files. Another impact of BitTorrent is that it allows for the distribution of bandwidth among many users and can alleviate hosting and bandwidth costs for users interested in making large files available.

BitTorrent has proven to be a hugely popular protocol since its inception. According to the BitTorrent website, BitTorrent has over 170 million unique users each month. ${ }^{83} \mathrm{~A} 2014$ report conducted by Sandvine noted that $24.53 \%$ of all daily upstream traffic in North America is due to BitTorrent, with $5.96 \%$ of all daily Internet traffic being accounted for by the protocol. ${ }^{84}$ In order to coordinate the servers that are uploading (seeding) and downloading (leeching) a file, a tracker is used. A tracker is a computer that coordinates the file distribution and often is represented on the Internet in the form of a web page with a user interface that allows for users to search the metadata about a torrent in order to find the file they are looking for. ${ }^{85}$

8I Wikipedia contributors, "Segmented file transfer," Wikipedia, The Free Encyclopedia, http://en.wikipedia.org/w/index.php?title=Segmented file transfer\&oldid=629|35424. http://en.wikipedia.org/w/index.php?title=Segmented_file_transfer\&oldid=629| 35424

82 "'Segmented File Transfer."

83 BitTorrent, "About BitTorrent," http://www.bittorrent.com/company/about.

84 Sandvine Intelligent Broadband Networks, "Sandvine Global Internet Phenomena Report IH 20।4," Sandvine Intelligent Broadband Networks, 6. https://www.sandvine.com/downloads/general/global-internetphenomena/20|4/Ih-20|4-global-internet-phenomena-report.pdf.

85 Bradley Mitchell, "What is a Bit Torrent Tracker?," About Technology (20 I4), http://compnetworking.about.com/od/bittorrent/f/bttracker.htm. 


\section{Public vs. Private Trackers}

There are numerous trackers on the Internet and they can be divided into two categories: public and private. Public trackers are readily available to users, do not require membership and usually are uncurated; in essence anyone can upload any file regardless of quality, copyright, or file format for users to download. Because these trackers are the most well- known and frequently used, they are often the source for much of the piracy that exists through BitTorrent. Their popularity also means that they are often the target of legal action and shut down due to copyright infringement. Frucci notes that trackers such as The Pirate Bay, Torrent Bay, Suprnova, and MiniNova have all been shut down due to legal actions. ${ }^{86}$ Private trackers differ from public trackers in that they are typically accessible only to members of the tracker and often have a specific content focus. Frucci notes the existence of a wide variety of private trackers for "music, for movies, for HD Blu-ray movie rips, for both Mac and PC software, for porn, for comic books, for console games, for anime, for TV shows, for E-books, and for sporting events." ${ }^{87}$ Private tracker communities typically contain highly devoted and active users, with strong subject knowledge and they are often highly curated with certain quality or content standards. For example, What.cd, a large music tracker, has certain quality standards for the audio files uploaded, and PassthePopcorn, a popular tracker devoted to cinema, has certain guidelines for what kinds of content can be uploaded.

There are no definite numbers on how many private trackers currently exist on the Internet. The blog Opentrackers.org provides a list of approximately 700 to 900 private trackers of varying sizes,; however, many of these trackers might not currently be active or have in fact been shut down. The reasons for shutdown typically involve the high costs of hosting and maintaining a tracker, which can range from monthly costs of $\$ 200$ to $\$ 1200$ or more depending on the size of the tracker ${ }^{88}$ Because most trackers do not charge for membership, they rely almost exclusively upon user donations to pay for monthly costs. In addition, the larger and more active a tracker is, the more time consuming it is to maintain. Many private trackers rely on volunteers to manage the day to day functioning of the site. It should also be noted that private trackers are not immune to legal action despite their relatively lower profile. In a noteworthy case, OiNK.cd, one of the leading private trackers dedicated to rare and hard to find music, was shut down in 2007 after investigation by the anti-piracy units of the International Federation of the Phonographic Industry and the British Phonography Industry. ${ }^{89}$ Despite cases like this, the specialized focus and smaller scale of many private trackers along with their restricted membership has allowed these sites to remain somewhat under the radar of anti-piracy organizations.

\section{Examining a Private Tracker Community}

As part of our research, we focused on a particular private tracker community dedicated to arthouse and classic cinema, as well as music and books, of which both the authors are members. In order to protect the identity of the users and administrators of the site, we shall not provide the name of the tracker, which we will refer to as $B C$ throughout this paper. BC is a leading private tracker started in 2005 that has grown to become a very popular and influential source for moving image content dating to cinema's infancy. Currently, the site has approximately 30,000 registered users and is host to nearly I50,000 torrents, of which about $60 \%$ are video files. In the nine years since its inception, the total traffic has totalled over 15,000 TB including both uploading and downloading.

86 Adam Frucci, “The Secret World of Private BitTorent Trackers," Gizmodo (February 19, 20 I0), http://gizmodo.com/5475006/the-secret-world-of-private-bittorrent-trackers.

87 Frucci, "Secret World."

88 Enigmax, December 14, 2009, "Private BitTorrent Trackers Commit Suicide With Rising Costs," Torrent Freak, https://torrentfreak.com/private-bittorrent-trackers-commit-suicide-with-rising-costs-09 | 2 | 4/.

89 Ernesto, October 23, 2007, "'Oink.cd Servers Raided, Admin Arrested," Torrent Freak, https://torrentfreak.com/oinkcd-servers-raided-admin-arrested//. 
Perhaps the greatest appeal of the site and the community is the ability to access hard to find material. The site is home to a wide range of content including rare silent gems such as Ernst Lubitsch's seldom seen and generally unavailable 1924 silent film, Forbidden Paradise. The film has never been released on any home video format and the version uploaded here contains only Czech flash intertitles, but given Lubitsch's stature and the complete lack of availability of the film otherwise, access to a subpar copy is still of great value. The site is also home to foreign cinema including the rare educational films of Iranian director, Abbas Kiarostami and alternate versions of established classics, such as the five-hour version of Serbian filmmaker Emir Kusturica's 1995 film Underground, which only aired once on German television and was never intended for wide release. The quality of the content on the site is generally quite high, with users encouraged to upload the highest quality files, often ripped from commercial Bluray releases or from other HD sources. While numerous low quality rips, often sourced from bootlegs of unknown origin, find a home on the site, their presence is primarily due to the unavailability of higher quality versions.

\section{Content Access and its Impact on Cinema Studies and Research}

Private trackers like $\mathrm{BC}$ are providing access to previously unavailable films from around the world and from every era and movement in cinema's history. lordanova argues that the "multitude of diaspora-driven Web vaults" 90 along with many other channels for streaming and downloading "are profoundly changing the landscape for the study of film." lol lordanova also suggests that the increasing availability of niche and "peripheral cinemas" 92 online takes knowledge of these texts out of the exclusive realm of subject experts and places it in the hands of anyone who has an online connection and a desire to watch the films. This allows for new avenues of research to be explored, both through making the cinema of virtually any movement, genre, director, or era available and allowing many different voices to participate in the discussion.

The utility of a private tracker like BC allows users to search for content by year, country of origin, genre, director or keyword. This allows unique and unexplored connections to be made by users, for example one can download an early MGM-talkie like 1930's The Big House and also download the foreign language versions (Revolte dans la prison and Menschen hinter Gittern) of the film that were produced at the same time. This could be useful to do a stylistic comparison of the three versions of the film, or alternately as research into the work of Hungarian auteur Pál Fejös, who directed the foreign language versions, or more generally as a jumping off point into research about foreign language versions of Hollywood films. The potential for new avenues of research grows every day as new content is made available.

\section{Why Does This Community Work: Crowdsourcing and Curation}

It is worth discussing how and why this content is made available on a private tracker like $\mathrm{BC}$. Once a person joins the tracker, they are free to begin participating by downloading and uploading content. On public trackers, it is common for users to download a torrent and, once the file is downloaded, to close the torrent and no longer upload the completed file to save their own bandwidth. In the public context, there is no incentive to upload content and no penalty for not seeding a file. In a private tracker community, this is not a viable strategy and in order to encourage users to upload new content and seed existing content, the concept of share ratio is used. A share ratio is determined by dividing the amount of data a user has uploaded by the amount a user has downloaded. Many private trackers set a minimum share ratio that users must maintain to keep their accounts in good standing. The ratio has the dual effect of incentivizing the uploading and seeding of content. For example, a user might upload a DVD-R file that is four gigabytes or more, or be inclined to download and seed a larger file that

90 Dina lordanova, "Instant, Abundant, and Ubiquitous: Cinema Moves Online," Cineaste, 39:I (Winter 20। 3 ): 46.

91 lordanova, "Instant, Abundant, and Ubiquitous," 46.

92 Iordanova, "Instant, Abundant, and Ubiquitous," 46. 
has just been uploaded by someone else in order to improve their ratio. This is in essence the currency of $\mathrm{BC}$ and private trackers, as the more content a user uploads and seeds, the more a user can then download. This has proven an effective tool for BC to stimulate the growth in content and to ensure that older content remains available through users seeding the files.

This idea of a collection sourced entirely by users fits neatly within the online model of crowdsourcing, which has been defined as the "the practice of obtaining needed services, ideas, or content by soliciting contributions from a large group of people and especially from the online community." ${ }^{33} \mathrm{~A}$ collection like that on BC could only be facilitated through crowdsourcing, as the breadth of the collection and sheer volume of data would be impossible for a single individual or organization to manage. For example, the time needed to upload a torrent (which can include ripping the file from a DVD or other source, creating a torrent file, providing screengrabs from the rip, creating metadata about the rip, as well as providing information about the film) can take 15 minutes to several hours. In addition, by drawing upon users from around the world to provide content, the collection becomes diversified in a way that transcends national boundaries and gives representation to potentially marginalized films, genres, or movements.

This notion of crowdsourcing the collection extends even further to aspects of curation as well. Users provide descriptions of content that can range from a brief synopsis to detailed cast lists, historical reviews, and other information about a film. In addition, users also upload images including historical posters and stills or add comments. Site members also have the opportunity to request a film to be uploaded and offer some of their ratio to the user who fulfils the request. This dynamic of sharing ratio provides a reward to users for helping others and also strengthens the collection and the community. The site also has monthly themes in which users are offered bonus ratio to upload content related to a certain director, genre, era, or region. Past themes include "Yugoslavian Cinema Under Tito","Joan Blondell”, and "Cannibal and Zombie Movies". These monthly films help to diversify the collection and highlight different aspects of the collection.

Perhaps the most powerful and unique aspect of BC's crowdsourcing and curation dynamic is the collaborative efforts of users to provide custom subtitles for films not available in English. The creation of custom subtitles is a time consuming process and is in many cases the only solution to being able to watch and understand a film when the viewer does not understand the language of the film. In many cases, films that for decades were unavailable in English have now been given English subtitles. Jean Renoir's 1932 talkie, La nuit du carrefour, one of the lesser-known films in the director's oeuvre, was given custom English subtitles by a BC user and can now be enjoyed by a larger audience. My favourite example of these subtitling activities pertains to famed French silent film director Louis Feuillade's eight hour serial Tih Minh from 1918. The version initially uploaded to BC had French and Dutch intertitles, but did not have English subs, however that did not impede one user from translating the film, despite not speaking either French or Dutch. In a March 2010 post on the Criterionforum.org message board, user swol7 recounts how he created the custom subtitles for the film:

\begin{abstract}
"So, with a friendly online French->English and Dutch->English translator in hand, I spent the last week and a half gradually going through the film, meticulously typing in the contents of each intertitle card (every last accent and cedilla!), sometimes in both languages, making the most sense out of them that I could, and crafting English subtitle files out of all of this."
\end{abstract}

This almost absurd level of commitment is emblematic of what Gilbert describes as the contemporary cinephile "who is savvy about circumventing...obstacles to make it possible...to view films that are otherwise unavailable... [through the] extra-legal downloading of films, or the

93 Merriam-Webster, s.v. "Crowdsourcing," http://www.merriam-webster.com/dictionary/crowdsourcing. 
process of making custom subtitles for un-translated works." 94 The cinephile audience that uses private trackers such as BC makes up a large and crucial force, driving much of the activity on the site, which in conjunction with the ratio mechanism allows the site to avoid stagnation.

\section{How Do Community Members See Themselves?}

As part of our research about BC, we surveyed users of the site to get a sense of their perception of who is using the site and why it is such a powerful tool. One respondent stated that the power of the site lies in its ability to allow users from around the world to have access to films previously available only to those in major film centres such as New York City. Another suggested that users of the site include casual film fans, collectors, cinephiles, and academics. One user identified himself or herself as a film programmer and noted that the content they downloaded from the site influenced their choices in what films they screen for the public. The potential for cross-pollination, where content from a private tracker is then made accessible in other formats, such as screenings, commercial DVD releases, or streaming is quite significant and remains underexplored. Some users we surveyed also pointed to the preservation role of the private tracker, suggesting that the digitization of films only available on formats such as VHS (and by doing so in the highest quality possible), qualifies as an act of preservation.

\section{Private Trackers as Preservation Tools?}

It is helpful to consider the digital preservation actions of the private tracker in relation to traditional notions of what constitutes film preservation. The U.S. National Film Preservation Foundation defines film preservation as "the full continuum of activities necessary to protect the film and share the content with the public." ${ }^{95}$ With traditional archives and cultural heritage institutions often lacking the resources to make content widely accessible, one must consider that these organizations may be very successful at ensuring the long -term safety of a film print, but are falling short in terms of sharing the content with the public. Conversely, a private tracker such as BC has no capacity to preserve physical objects, but is an incredible tool for sharing digital objects. Through the acts of downloading and uploading, these objects are duplicated, disseminated, and stored on hard drives and servers around the world, which helps to ensure the potential for long-term preservation of each object.

\section{The Development of Sharing Networks}

The range of activities conducted within torrent communities, and the evolution of the sharing infrastructure involved, have progressed in recent years in tandem with developments elsewhere; from improvements in software and hardware to broader, infrastructural advances in internet services, all occurring in step with an ever growing base of users, supporting and contributing to repositories. The inchoate digital film archive is not standardised, nor its future safeguarded, but nonetheless it has developed, organically and incrementally, into a relatively concrete form. Attempting to assess the health of torrent communities online is complicated, not only by the limitations on access and visibility imposed by trackers but by the fluctuating shape of the online ecosystem, in which even sites that are - per Frucci's analysis ${ }^{96}$ - "closed" may still effectively provide service - whether through new incarnations, their domain suffixes affiliated with more permissive territories; through dormant, cached links to content hosted diffusely; or in the reconstitution and circulation of their holdings under new protocols and in new contexts.

Among the factors dictating the shape of the contemporary archive, one at once present and conspicuously absent is copyright law. A general analysis of the legality of these archives un-

94 Andrew W. Gilbert, “Gender, Taste, and Cinephile Culture," (PhD diss., Roosevelt University, 20 I3), 23. http://search.proquest.com/docview/I356849369.

95 National Film Preservation Foundation, The Film Preservation Guide:The Basics for Archives, Libraries, and Museums, (San Francisco: National Film Preservation Foundation, 2004), 4.

96 Frucci, "Secret World." 
der American copyright law is relatively straightforward, informed by U.S. Code Title 17 and affirmed by the results of file-sharing litigation unfolding since the turn of the century. The distinct activities involved in circulating copyrighted works online without the permission of a rights-holder - as an uploader; a downloader; or as a third party, facilitating these transmissions - each violate various protections afforded to the rights-holder under the act (the right to reproduce the work; to prepare derivative works; to distribute copies; to perform or display the work publicly ${ }^{97}$ ), applicable to a vast majority of artistic works created. While caveats to the act regarding the "fair use" of material - generally undefined enough to allow for some interpretation as to what might constitute such use ${ }^{98}$ — present exemptions from strict compliance with restrictions on reproduction and display, it is reasonable to state that a straight reading of the act would find (and repeatedly has found) many instances in which obtaining a film online would be a breach of the law. With reference to the proportion of work covered by regulation, writer James Boyle has claimed that $95 \%$ of the culture created in the twentieth-century is protected by copyright provisions that make it unavailable, ${ }^{99}$ whether because it is out of print, out of circulation, or still tied to licensing agreements preventing its reproduction.

As such, in considering the legal situation of the online film repository, it is perhaps more valuable to consider the law as it is practiced, rather than written. The infringements described above have usefully been described as "low-risk violations," a term conceding that such activity violates copyrights, while acknowledging the pragmatic obstacles limiting consequences for users. The strategies employed in recourse to copyright violation in the internet era have varied in scale and efficacy, evolving in response to the various techniques developed to avoid detection; in reference to the particular activities associated with circulating 'cinephile' content online, one significant factor influencing the relatively modest risk is the limited immediate commercial value of the holdings circulated, making pursuit of violators a concern secondary to the attention focused on large-scale piracy of studio productions.

More generally, the nature of digital content has highlighted the inadequacy of litigation as an effective means through which to respond to piracy. The labour and considerable expense involved in drafting a cease and desist letter - which might succeed in targeting a specific distributor of a pirated work, but which would not address subsequent circulation of the work, incompatible with the various scales and territories in which this occurs - has often proved equivalent in effect to action taken informally, whereby a rights-holder's wish that content be removed is expressed, but without the explicit threat of legal action. The consequent suppression of content stolen or liberated from the archive is best measured not by its availability or unavailability, but by the degree of ease involved in locating it - whether it can be retrieved promptly through a Google search, or is restricted to clandestine quarters, requiring registration, membership or extensive navigation to find it.

\section{I0. Stepping Out of the Shadows}

It is valuable, then, to assess whether the needs that private digital repositories meet might also be met outside of the conflicted terrain they currently inhabit - illegal but widely used, circulating material but subject to restrictions. Two enterprises pertinent to this assessment, and worthy of comparison here, are the Internet Archive and UbuWeb.

Archive.org, the Internet Archive, is the non-profit archive "working to prevent the Internet ... and other "born-digital" materials from disappearing into the past"; its myriad projects encompass significant collections of material, across varied media, chronicling cultural activity,

97 "Copyright Law of the U.S.A. and Related Laws Contained in Title 17 of the United States Code", United States Copyright Office, 20 I4, http://www.copyright.gov/title I 7/92chap I.html\# I-38.

98 "Copyright Law of the U.S.A. and Related Laws Contained in Title 17 of the United States Code", United States Copyright Office, 20 I4, http://www.copyright.gov/title I 7/92chap I.html\# I -40.

99 Voyce, Stephen. 20 II. "Toward an Open Source Poetics: Appropriation, Collaboration, and the Commons." Criticism 53 (3): 407-438. 
"from ephemera to artefact."100 Popular, visible projects hosted by Internet Archive projects, such as David Pierce and Eric Hoyt's Media History Digital Library, ${ }^{101}$ are designed to adhere strictly with the copyright terms of the material contained, representing only the parts of the collection that have passed into the public domain, in the case of the Media History Digital Library making available the issues of journals whose period of copyright protection has expired or was not renewed. ${ }^{102}$ Additional collections include user-contributed material and "orphaned" films, occupying a more complicated rights position. Since 20I2, the Internet Archive has operated two trackers, facilitating the distribution of more than five million items using the BitTorrent protocol. ${ }^{103}$

The website UbuWeb was established as a resource for avant-garde cultural material, growing to accommodate a variety of film and moving image content, historically having provided access to seminal work by artists like Samuel Beckett, Man Ray, and Nam June Paik. Considering the mandate of the site, and the copyright status of works held, founder Kenneth Goldsmith has said, "If it's out of print, we feel it's fair game. Or if something is in print, yet absurdly priced or insanely hard to procure, we'll take a chance on it." ${ }^{04}$ The content available exists in constant flux, its library growing but also, occasionally, changing; UbuWeb's mission to make out-of-circulation works available has in some cases sparked a conversation with rightsholders, in which permissions were negotiated in response to the objections of creators. One example of this is the site's selection of works by avant-garde Canadian filmmaker Michael Snow: Goldsmith recalls that Snow reached out to the site to ask that certain films hosted be removed, while approving the exhibition of others; per Goldsmith, "Having two permissioned films from Michael Snow beats hosting ten without his blessing." 105 UbuWeb's highly visible, public display of work previously restricted by copyright protections challenges the validity of those protections, accentuating their limitations as well as their values, interrogating whether automatic protection correlates with broader concerns regarding intentionality and access. The site is a compromise, boldly attempting to move works towards exhibition while cognisant of the opportunities and risks posed by this action.

Discussing the use of archival holdings within an institutional context, archivist and filmmaker Rick Prelinger has described access as "overwhelmingly crippled by an overzealous application of the precautionary principle." 106 Attempting to effectively navigate the varied gray space around copyright presents challenges to already under-resourced archives, whereas embracing straightforward adherence to the strictest interpretation of the law is good policy, negating the risk imposed by potentially ruinous punitive measures. Generally, both under the law and in practice, the individual circumstances of an item or collection will dictate the feasibility of sharing it more widely. However, we believe that it is important to recognise the new reality under which much copyright-protected content within the archive exists: a great many restricted items are no longer truly "unavailable", per the terms of their copyright, but instead are available selectively, either to those with establishment affiliation or credentials, or to those both literate and privileged enough to be in a position to navigate unofficial channels to retrieve derivative copies. The example of UbuWeb is instructive, as much for its dialogue with featured artists as for its partnership with institutions like WFMU and PennSound at the University of Pennsylvania, contributors of "technical, moral, and spiritual" support,"107 making possible the site's mission. Between the conversational, activist model of UbuWeb, and the Internet Archive's position as the vanguard of

\footnotetext{
100 "Internet Archive: About IA", Internet Archive, 20 I4, http://archive.org/about/.

I0I Media History Digital Library: Free Texts : Download \& Streaming : Internet Archive, Internet Archive, 20 I 4, https://archive.org/details/mediahistory.

102 Media History Digital Library - Online Access to the Histories of Cinema, Broadcasting \& Sound, Media History Digital Library, 20 I 4, http://mediahistoryproject.org.

103 Internet Archive Frequently Asked Questions, Internet Archive, 20I4, https://archive.org/about/faqs.php\#320.

I04 U B UW E B :: Frequently Asked Questions, UbuWeb, 20 I4, http://www.ubu.com/resources/faq.html

105 Goldsmith, Kenneth. "An Open Letter to the Frameworks Community", UbuWeb, http://www.ubu.com/resources/frameworks.html.

106 Prelinger, Rick. 2007. "Archives and Access in the 2 I st Century." Cinema Journal 46 (3): I | 4 - I I 8.

107 "U B UW E B :: Partners", UbuWeb, 20 I4, http://www.ubu.com/resources/partners.html.
} 
digital archiving, the shape of a feasible and legitimate digital film repository begins to materialise - one that might provide access to material where copyright is deemed flexible, and under conditions sensitive to the context in which works are displayed. While acknowledging a tension that has long existed between rights owners and audiences, and recognising the complexity of the decisions faced by custodians, it is our hope that the infrastructures developed to circulate film material might be adopted and appropriated to facilitate broader access to the archive.

\section{I. Bibliography}

BitTorrent."About BitTorrent.” http://www.bittorrent.com/company/about.

Enigmax. "Private BitTorrent Trackers Commit Suicide With Rising Costs.” Torrent Freak (December 14, 2009). https://torrentfreak.com/private-bittorrent-trackers-commitsuicide-with-rising-costs-091214/.

Ernesto."Oink.cd Servers Raided, Admin Arrested.” Torrent Freak (October 23, 2007). https://torrentfreak.com/oinkcd-servers-raided-admin-arrested/.

Frucci, Adam. "The Secret World of Private BitTorrent Trackers." Gizmodo (February I9, 20 I0). http://gizmodo.com/5475006/the-secret-world-of-private-bittorrent-trackers.

Gilbert, Andrew W. “Gender,Taste, and Cinephile Culture.” PhD diss., Roosevelt University, 20 I3. http://search.proquest.com/docview//356849369.

Goldsmith, Kenneth. “An Open Letter to the Frameworks Community.” UbuWeb. http://www.ubu.com/resources/frameworks.html.

Internet Archive."Internet Archive:About IA.” Internet Archive, 20 I4. http://archive.org/about/.

Internet Archive.“Internet Archive Frequently Asked Questions.” Internet Archive, 2014. https://archive.org/about/faqs.php.

Internet Archive."Media History Digital Library : Free Texts : Download \& Streaming : Internet Archive." Internet Archive, 2014. https://archive.org/details/mediahistory.

lordanova, Dina."Instant, Abundant, and Ubiquitous: Cinema Moves Online." Cineaste, 39:I (Winter 20I3): 46-50.

Media History Project."Media History Digital Library - Online Access to the Histories of Cinema.” Broadcasting \& Sound, Media History Digital Library, 2014. http://mediahistoryproject.org.

Merriam-Webster, s.v. “Crowdsourcing," accessed November 29, 20I4, http://www.merriam-webster.com/dictionary/crowdsourcing.

Mitchell, Bradley. “What Is a Bit Torrent Tracker?” About Technology (20I4). http://compnetworking.about.com/od/bittorrent/f/bttracker.htm.

National Film Preservation Foundation. The Film Preservation Guide:The Basics for Archives, Libraries, and Museums, (San Francisco: National Film Preservation Foundation, 2004).

Prelinger, Rick. 2007. “Archives and Access in the 2 I st Century.” Cinema Journal 46 (3): I I 4- I I8. 
Sandvine Intelligent Broadband Networks. "Sandvine Global Internet Phenomena Report IH 20I4." Sandvine Intelligent Broadband Networks.

https://www.sandvine.com/downloads/general/global-internet-phenomena/20I4/lh2014-global-internet-phenomena-report.pdf.

UbuWeb.“U B UW E B :: Frequently Asked Questions.” UbuWeb, 2014. http://www.ubu.com/resources/faq.html.

UbuWeb.“U B UW E B :: Partners.” UbuWeb, 20I4. http://www.ubu.com/resources/partners.html.

United States Copyright Office. "Copyright Law of the U.S.A. and Related Laws Contained in Title I 7 of the United States Code." United States Copyright Office, 20 I4. http://www. copyright.gov/title I7/92chapl.html.

Voyce, Stephen. 20I I. “Toward an Open Source Poetics:Appropriation, Collaboration, and the Commons." Criticism 53 (3): 407-438.

Wikipedia Contributors. "Segmented file transfer." Wikipedia,The Free Encyclopedia.

http://en.wikipedia.org/w/index.php?title=Segmented file transfer\&oldid=629135424

\section{Images}
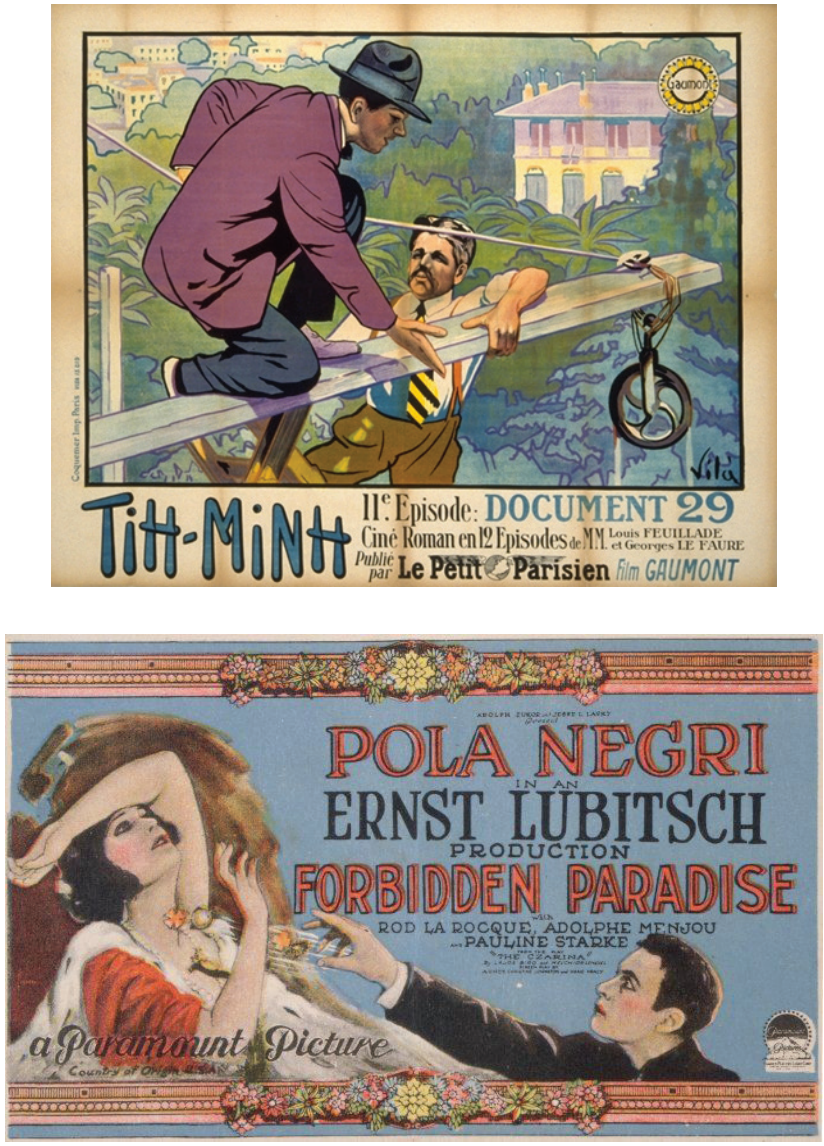